\title{
A ficção científica de acordo com os Futurians
}

\author{
Science Fiction According to the Futurians
}

Andreya Susane Seiffert ${ }^{1}$

DOI: 10.19177/memorare.v8e12021204-216

\begin{abstract}
Resumo: The Futurian Society of New York, ou simplesmente The Futurians, foi um grupo de fãs e posteriormente escritores e editores de ficção científica, que existiu de 1938 a 1945. 0 período é geralmente lembrado pela atuação do editor John Cambpell Jr. à frente da Astounding Science Fiction. A revista era, de fato, a principal pulp à época e moldou muito do que se entende por ficção científica até hoje. Os Futurians eram, de certa forma, uma oposição a Campbell e seu projeto. Três membros do grupo viraram editores também e foram responsáveis por seis revistas pulps diferentes, em que foram publicadas dezenas de histórias com autoria dos Futurians. Esse artigo analisa parte desse material e procura fazer um pequeno panorama de como os Futurians pensaram e praticaram a ficção científica no início da década de 1940. Palavras-chave: Ficção Científica. Futurians.
\end{abstract}

\begin{abstract}
The Futurian Society of New York, or simply The Futurians, was a group of fans and later writers and editors of science fiction, which existed from 1938 to 1945. The period is generally remembered for the role of editor John Cambpell Jr. at the head of Astounding Science Fiction. The magazine was, in fact, the main pulp at the time and shaped much of what is understood by science fiction until today. The Futurians were, in a way, an opposition to Campbell and his project. Three members of the group became editors as well and were responsible for six different pulp magazines, in which dozens of stories were published by the Futurians. This article analyzes part of this material and seeks to provide a small overview of how the Futurians thought and practiced science fiction in the early 1940s.

Keywords: Science Fiction. Futurians.
\end{abstract}

\footnotetext{
${ }^{1}$ Doutora em História Social (USP). Contato: bucaseiffert@gmail.com
} 


\section{A origem dos Futurians}

Na década de 1930 nos Estados Unidos, os clubes de fãs de ficção científica multiplicavam-se país afora e reuniam em sua maioria jovens entusiastas do gênero literário. Em 1938, um novo clube foi criado e batizado de The Futurian Science Literary Society. 0 neologismo Futurian foi retirado de uma fanzine inglesa com esse nome e se aproxima em português de algo como "futurano". Na quarta reunião, o nome do grupo foi modificado, e passou a ser The Futurian Society of New York, ou simplesmente The Futurians, termo indicado para falar também de seus membros. 0 grupo reunia nomes como Donald A. Wollheim, John B. Michel, Robert W. Lowndes, Frederik Pohl, Isaac Asimov, James Blish e Cyryl Kornbluth, além de outros que foram sendo incorporados ao longo dos anos, como Damon Knight e Judith Merril. Todos os membros eram bastante jovens, a maioria saindo da adolescência. Assim, seus anos de formação foram passados no grupo, e a noção de ficção científica foi construída em conjunto por eles, primeiro como fãs e leitores, e depois também como escritores, editores e ilustradores.

No começo, The Futurian Society of New York se assemelhava aos demais grupos de ficção científica à época, e se reunia uma ou duas vezes por mês, muitas vezes na sede da liga comunista juvenil (YCL, em inglês), da qual vários membros faziam parte. Já no ano seguinte a sua criação, no entanto, o grupo começou a perder a rigidez comum aos clubes de então. The Futurian Society foi se transformando em uma espécie de família estendida. Tanto é que vários membros passaram a morar junto, sendo a Ivory Tower, ou torre de marfim, a mais famosa dessas moradias compartilhadas.

A discordância com membros de outros grupos contribuiu para que os Futurians, de certa forma, se fechassem entre si. Essa discordância vinha já desde antes da fundação do grupo: em 1937, em uma convenção de fãs de ficção científica, Donald Wollheim leu um discurso escrito por John Michel em que ele argumentava que o gênero deveria se engajar com as questões de seu tempo. Isso gerou um grande debate e dividiu o fandom entre aqueles que concordavam ou discordavam das ideias de Michel. Os favoráveis à ideia organizaram-se em um movimento que ficou conhecido como "Michelismo" em referência a Michel e seu discurso. Os "michelistas" promoveram a distribuição de panfletos, o envio de cartas para as revistas pulp pedindo artigos de ciências sociais e a criação de uma fanzine.

Também em 1937, John W. Campbell Jr. assumiu como editor da revista Astounding Science Fiction e muitos consideram os anos seguintes como "a época de ouro" da ficção científica, justamente pela influência de Campbell:

Campbell and his magazine Astounding stand for the second era of the sf magazines as Gernsback stands for the first. The period that begins with his editorship is often called the Golden Age of sf, and many of the bestknown writers in the field first appeared in his magazine (ATTEBERY, 2003, p. 67).

Isso tende a apagar outras contribuições, como é o caso dos Futurians. Roger Luckhurst, no entanto, lembra que eles eram uma força 
tão importante quanto Campbell à época da Feira Mundial de Nova Iorque (1939):

Just before the fair began, two of the most important groupings in American SF developed in New York. Both believed that American SF could be transformed from its juvenile and pulp origins into a serious and committed literature; each had very divergent views on what that commitment might be (LUCKHURST, 2005, p. 66)

Como apontado por Luckhurst, as ideias acerca de como deveria ser a ficção científica divergiam bastante entre Campbell e os autores ligados à Astounding e os Futurians. Há centenas de trabalhos a respeito de Campbell e da Astounding, mas os Futurians ainda são pouco estudados. Assim, esse artigo procura montar um pequeno panorama mostrando como os Futurians pensavam e praticavam a ficção científica.

\section{Os Futurians nas pulps}

Quando o grupo The Futurian Society of New York foi criado, em 1938, muitos dos seus membros eram "michelistas" e acreditavam que a ficção científica deveria se posicionar politicamente. 0 "michelismo" perdeu força depois de alguns meses, mas foi uma importante intersecção da ficção científica com a esquerda americana, conforme Sean Cashbaugh:

Though a minor movement in both the histories of SF and the American Left, and one that was ultimately a failure, Michelism was a crucial intersection of these histories and as such highlights the radical possibilities of forms, genres, and communities often considered marginal to leftist literary activity (CASHBAUGH, 2016, p. 66).

É possível encontrar ecos do "michelismo" em alguns contos publicados pelos Futurians, especialmente os escritos por Michel, ainda que o movimento já não estivesse em voga quando os Futurians passaram de fãs a produtores de ficção científica. A transição se deu em um período em que as revistas pulp de ficção científica viviam um boom no mercado editorial, e eles fizeram parte desse momento.

A primeira revista pulp de ficção científica dos Estados Unidos foi a Amazing Stories, criada em 1926 por Hugo Gernsback. Somente em 1939, nove novas pulps de ficção científica foram lançadas. Nos dois anos seguintes houveram ainda novos títulos lançados e, incluindo os que já existiam anteriormente, era possível encontrar em 1941 vinte e duas revistas pulps diferentes dedicados ao gênero (ASHLEY, 2000).

O primeiro Futurian a se tornar editor foi Frederik Pohl, com apenas dezenove anos de idade. A Popular Publications contratou Pohl para ser o editor de duas novas revistas de ficção científica: Astonishing Stories e Super Science Stories, lançadas em fevereiro e março de 1940, respectivamente.

Já a Albing Publications concordou em lançar duas pulps de ficção científica, Stirring Science Stories e Cosmic Stories, tendo o Futurian Donald Wollheim como editor. A editora, no entanto, não tinha orçamento disponível e eles fizeram um arranjo em que se as revistas tivessem sucesso, Wollheim passaria a receber um pagamento como editor e uma verba para comprar histórias após a terceira edição delas. Para conseguir entregar duas revistas sem dinheiro para poder pagar 
pelas histórias, Wollheim contou com os colegas do grupo. As primeiras edições das revistas, com datas de fevereiro e março de 1941, continham quase que exclusivamente histórias dos Futurians.

O Futurian Robert Lowndes assumiu o comando das pulps Future Fiction (depois rebatizada de Future Combined with Science Fiction Stories) e Science Fiction Quarterly, da Columbia Publications. Os primeiros números editados por Lowndes foram publicados em abril de 1941:

Under Lowndes's editorship Future Fiction and Science Fiction Quarterly were transformed. This was because Lowndes was able to rely upon his close colleagues in a fan organization known as the Futurians (...). In fact the depth of talent in the Futurians provided a most welcome breath of fresh air to science fiction. Outside of the writers that Campbell was developing at Astounding, they were the most creative force in science fiction (ASHLEY, 2000, p. 149).

De acordo com Mike Ashley, a participação dos Futurians nas revistas pulp trouxe frescor para a ficção científica de então. Os três editores do grupo controlaram, ao todo, seis publicações dedicadas ao gênero. Eles puderam inovar e publicar histórias que eles, enquanto fãs, gostariam de ler nas revistas. Além disso, os outros Futurians tiveram um espaço para publicar seus textos e assim se aperfeiçoar enquanto escritores, além de permitir que eles explorassem e experimentassem novos temas e abordagens.

A maior parte das histórias publicadas pelos Futurians no início da década de 1940 se deu nas seis revistas editadas pelos membros do grupo, embora vez ou outra publicassem também em outras pulps. A exceção fica por conta de Asimov, que preferia publicar seus textos na revista "rival", a Astounding de Campbell, por ser a pulp de mais prestígio e também a que melhor pagava os autores - um centavo por palavra; já Pohl, por exemplo, podia pagar apenas meio centavo por palavra para os autores das histórias que comprava (POHL, 1978). Quando Wollheim iniciou como editor e pediu aos colegas que doassem histórias para suas revistas, Asimov topou. Depois, no entanto, ouviu um editor falar que isso era concorrência desleal e ficou preocupado que Campbell nunca mais comprasse uma história dele se soubesse que ele participou desse esquema. Então ele pediu ao Wollheim um pagamento, qualquer que fosse, o que deixou Wollheim muito irritado. Ele mandou um cheque de cinco dólares e uma nota desaforada dizendo que a única coisa de valor na história era o nome do Asimov e que assim ele estava recebendo $\$ 2,50$ por palavra (ASIMOV, 1979). A história chama "O sentido secreto" e nela um humano fica triste porque um extraterrestre não tem os sentidos tão desenvolvidos quanto dos humanos, mas acaba descobrindo que eles tem outros sentidos que nós não temos.

Uma prática que começou na Astonishing e se estendeu por muitos anos foi a escrita em conjunto. Na edição de abril de 1940, Pohl estava com um buraco de 10.000 palavras, que os colegas se dispuseram a preencher. Richard Wilson e Cyril Kornbluth fizeram uma versão inicial, que Dirk Wylie "homogeneizou" (RICH, 2009). A noveleta ganhou o título de "Stepsons of Mars" e traz a descoberta de um metal em Marte que é a cura para o câncer na Terra. Marte, no entanto, é ocupada por estranhos alienígenas. 0 pseudônimo usado pelo trio para publicar 
"Stepsons of Mars" foi "Ivar Towers", em referência a "Ivory Tower", nome da moradia coletiva.

Mark Rich narra um episódio curioso envolvendo o grupo e que novamente mostra a dinâmica que havia entre os Futurians:

A new magazine was being launched that winter by H-K Publications. Its name was to be Comet Stories, and its editor was no less than the former editor of Astounding, F. Orlin Tremaine. That it would be paying the standard rate of a penny per word came as music to writers' ears. One such hopeful, Don Wollheim, submitted a story entitled "The Psychological Regulator" in hopes of finding his place within the new pulp title. It came back from Tremaine, however. In a round-robin process unlikely to have occurred in any group besides The Futurians, Doc Lowndes then rewrote it, submitted it to Comet, and received his rejection. Michel tried his hand, to the same result, after which Elsie Balter then took her turn (RICH, 2009, p. 72).

Cyril Kornbluth resolveu tentar também e sua versão foi aceita por Tremaine, e ele dividiu os lucros da história com Wollheim. Na história, uma máquina é usada para "ajustar" problemas mentais. Um médico acaba por programar ela em um formato antigo e transforma seu paciente em um viking, que resolve tomar a cidade. 0 episódio da reescritura de "The Psychological Regulator" mostra como a produção da ficção científica não era vista pelos Futurians como um ato isolado, individual, mas sim que poderia ser feito de forma coletiva. Cada leitor da história de Wollheim transformou-se num autor, construindo uma nova versão daquilo que leu.

"The Martians are Coming", publicada no primeiro número da Cosmic Stories, é outro exemplo de escrita em conjunto. Embora na revista o conto apareça atribuído a Robert Lowndes, os colegas Cyril Kornbluth e Donald Wollheim ajudaram a escrever a história. Em "The Martians are Coming", dois jornalistas arrecadam dinheiro para construir uma máquina para se comunicar com Marte. A história é bastante engraçada, e faz referência a vários elementos da ficção científica. Quando conseguem ver uma cidade marciana, eles comentam: "Very ordinary, I think", said Whitlowe. "Seems as if you're right. Frank R. Paul would be horribly disappointed" (LOWNDES, 1941, p. 38). Frank R. Paul era um famoso ilustrador de ficção científica e que produziu diversas capas para a Amazing Stories, a primeira pulp de ficção científica. Vários textos dos Futurians faziam essa espécie de jogo com os leitores, remetendo a nomes da ficção científica ou a histórias antigas. Assim, os Futurians conectavam essas narrativas e ajudavam a construir a história da ficção científica nas próprias histórias.

"The Martians are Coming" é baseada na transmissão de "Guerra dos Mundos" feita por Orson Welles (RICH, 2009). No caso da história escrita pelos Futurians, os marcianos não são seres perigosos e violentos que querem se apossar da Terra. Os marcianos contam para os jornalistas que sofrem com a falta de água. Os dois, bêbados, convidam os marcianos para virem morar na Terra. Depois, se dão conta do que fizeram, procuram o governo e falam que foram hipnotizados e que os marcianos estão vindo invadir a Terra. 0 mundo, então, se une de uma forma inesperada e inédita; guerras em curso são canceladas e todos os esforços são voltados para a "ameaça" marciana. Os marcianos avisam que vão pousar em New Jersey, assim como na transmissão de Welles, e 
toda a frota vai para lá; as cidades próximas são evacuadas. Os marcianos enviam uma mensagem dizendo que pousaram e que estão sendo atacados, mas ninguém viu sinal de suas naves. Um dos jornalistas, então, percebe um movimento de insetos próximo de onde eles estão:

It was a turbulent knot of insects, distinguished by bluish flashes of light. Whitlowe lowered the beam to the ground below. There were arrayed the two thousand ships - tiny things, about the size of cigarettes.

"And that," said Gary, "is the Martian race. All bets are off, and, if we wish to save our insignificant but witty friends from the monstruous gnats and mosquitoes that are beseigning them, we'd better rush out some Flit." (LOWNDES, 1941, p. 45).

0 conto se encerra assim, de maneira bem-humorada. A ilustração da história foi feita pelo também Futurian David Kyle e simula uma capa de jornal, referência ao pânico noticiado pelos jornais após a transmissão de Welles. É importante destacar que as ilustrações nas revistas eram uma peça fundamental da ficção científica à época, sendo consideradas como parte dos textos pelos leitores:

Figura 1: ilustração do conto "The Martians are Coming"

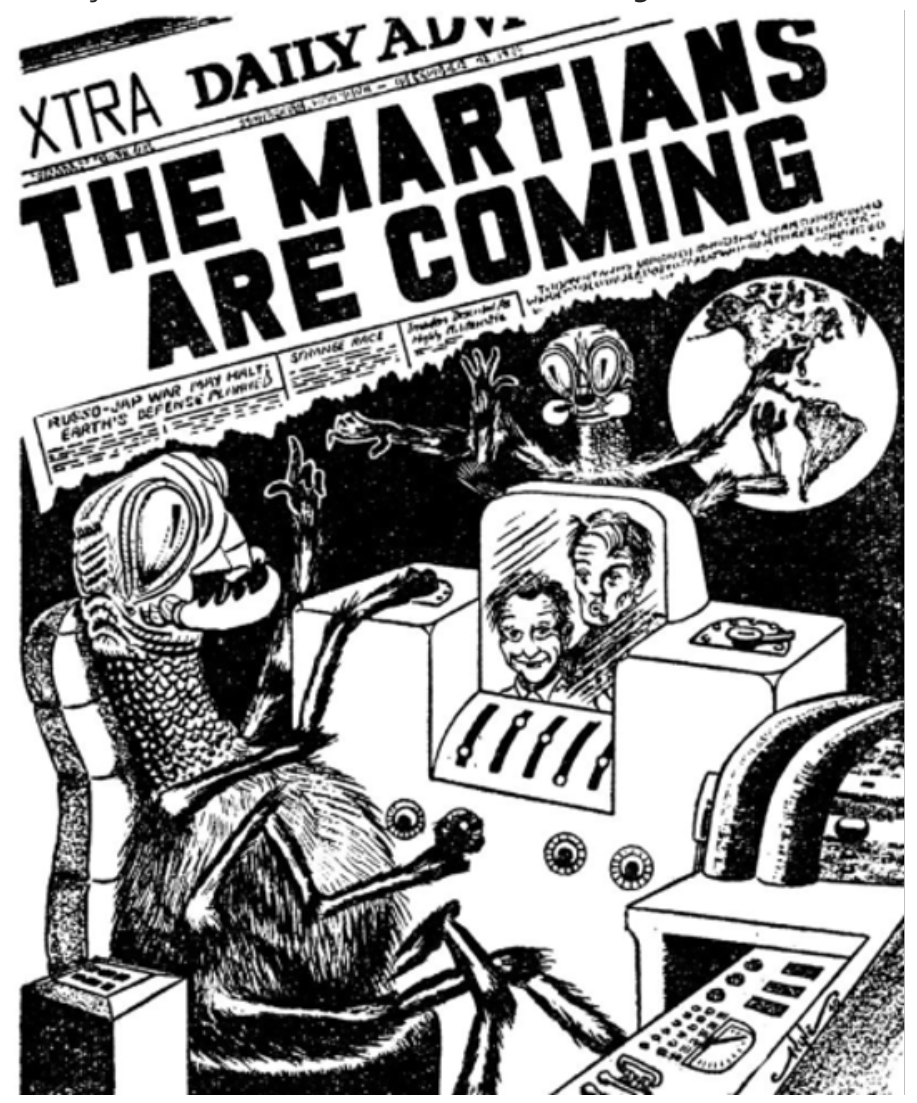

Fonte: Eaton Collection, fotografia da autora

Se Wells usou os marcianos como metáfora para o imperialismo britânico e Welles para as invasões nazistas (DAVIS, 2018; VINT, 2014), os Futurians não pareciam querer discutir nenhuma questão geopolítica com "The Martians are Coming". Ainda que se possa pensar o conto a partir de uma perspectiva do encontro com o outro (e como esse outro é um reflexo dos nossos medos), a questão principal me parece ser o 
próprio gênero literário. Com suas referências internas, "The Martians are Coming" é uma ficção científica que procura rir de si mesma.

Ao se lançarem como escritores, os Futurians procuraram circular entre os gêneros, criar algo novo, experimental. 0 grupo tentou desmontar algumas noções antigas sobre a ficção científica. Para eles, tratava-se de literatura, e como toda narrativa, era possível fazer diversos experimentos com ela. Ela não precisava ter, necessariamente, um propósito. Algumas vezes, como no caso de "The Martians are Coming", era suficiente apenas entreter os leitores.

Pensando em se aprimorarem enquanto escritores, os Futurians criaram uma espécie de seminário de escrita dentro do grupo, conforme relatou Robert Lowndes para Damon Knight:

The Cabal was a literaray workshop, really. We met at Cyril's place once a week, and each one of us was supposed to bring a manuscript to be read (...). And as it turned out, a fair number of manuscripts that were read at the Cabal were later sold and published (...). If either Donald or myself felt a story was good enough to use, we'd accept it on the spot (KNIGHT, 2003, l.1462).

Além dos seminários acerca de escrita e também das centenas de conversas informais, os Futurians também procuraram ampliar esse debate com os demais fãs. Um exemplo interessante ocorreu em 1941, na edição de agosto da Future Fiction. Robert Lowndes, que era editor da pulp, escreveu o ensaio "Science Fiction Classics" sob o pseudônimo de Wilfred Owen Morley. Nele, ele se perguntava sobre o que faria uma obra de ficção científica se tornar um clássico. Depois de recusar a precisão científica e a profecia, ele conclui:

And if, in the 21st, $22 \mathrm{~d}$ and $23 \mathrm{~d}$ century, any of the science fiction stories you read today will be read with interest and enthusiasm then, it will be because the readers of that distant future think, as they peruse the stories in question: "This person had a remarkably modern viewpoint. Why, I can't believe that he really lived in those terrible days of the 20th century" (MORLEY, 1941a, p. 95).

Na edição de dezembro da Future Combined with Science Fiction (novo nome da revista), foi publicada uma carta de J.S. Klimaris (pseudônimo do Futurian Walter Kubilius) discordando do ensaio:

Science fiction can become classic only when it becomes living literature. All literature, dear Morley, is alive when it is an emotional expression of human experience or human aspiration. Science fiction can become living literature when if fulfills that requirement. What true science fiction reader has not wept with Mary Shelley's monster that tried to win the love and understanding of Dr. Frankenstein? Who has not felt a lump in his throat as Nemo's Nautilus finally sank beneath the waves after defying mankind and twenty thousand leagues under the sea? Who has not looked uneasily up at the strange stars after the War of Worlds ended? Who has been able to sail calmly over a quiet sea after Poe's "Descent into a Maelstrom"? Who among us is not a Faust or a Manfred? In the light of such living literature, and they are also science fiction classics, Morley's words about "modern outlook" fade away into nothingness (KLIMARIS, 1941, p. 67).

Lowndes, assinando como Morley, respondeu logo abaixo a carta, dizendo que talvez ele tenha escolhido o termo errado, e que 
"universal", "atemporal" ou "progressista" teria sido melhor. Ele continua:

These stories which you mention in your last paragraph all have the "timeless" quality to which I refer. They are not bound up in the very local and limited concepts of any given period, even though the writing may, in some cases, be archaic. What I meant by "modern outlook" was this: read any of these examples of "living literature" and you do not get the feeling of being cramped into the obsolete concepts, superstitions, conceits, etc., of a long-dead age. There is something in them that will be new and "modern" so long as homo sap remains as he is, essentially (MORLEY, 1941b, p. 68).

Essas cartas demonstram que os Futurians estavam o tempo todo refletindo, debatendo as obras que consideravam as clássicas do gênero e tentando, juntos, construir uma ficção científica. A forma como pensavam que o gênero deveria (ou não) ser tem a ver com que tipo de literatura buscavam construir. Para eles, o que estava em jogo não era (tanto) a relação da ficção científica com a ciência, mas sim com a própria ficção.

Um outro exemplo muito interessante da construção coletiva da ficção científica aconteceu nas mesmas edições em que Lowndes e Kubilius debatiam sobre o gênero. A história "A Million Years and a Day" foi escrita pelo Futurian Donald Wollheim e publicada na edição de agosto de 1941 da Future Fiction. No conto, dois astronautas - um homem e uma mulher - voltam para a Terra e encontram toda a humanidade suspensa por uma espécie de coma. 0 mundo estava dividido por uma guerra e os dois lados criaram uma arma que, quando acionada, deixava a outra metade dormindo. Os astronautas descobrem que há dois botões possíveis de serem acionados na arma: se eles acionarem a opção "renovação" da arma, todos que estão dormindo morrerão. É possível, no entanto, acordar metade ou mesmo toda a humanidade - só que se fizerem isso é provável que a guerra continuará. 0 homem quer apertar o botão renovação e propõe que os dois recomecem a humanidade. Já a mulher não tem certeza, mas é ela quem aperta um botão. A história termina com o final aberto, sem saber o que ela decidiu. 0 editor da revista então, o Futurian Robert Lowndes, pede sugestões aos leitores do que é que aconteceu. Janice apertou os botões para renovação, matando toda a humanidade? Ou foi para acordá-la e assim dar continuidade à guerra? Como forma de incentivo para que os leitores enviassem suas respostas, é anunciado que as três melhores irão ganhar as ilustrações originais daquele número da revista.

O concurso revela que questões os Futurians consideravam que a ficção científica deveria tratar. Para além de extrapolações científicas ou literatura escapista, há outras reflexões possíveis. No caso de "A Million Years and a Day", um dilema moral. Cabia ao leitor colocar-se no papel de autor e pensar um desfecho para a história. 0 exercício de imaginação proposto tanto pelo autor quanto pelo editor da revista oferecia um papel ativo para os leitores na elaboração da história. Dessa forma, a ficção científica era pensada de forma conjunta e experimental e construída o tempo todo no próprio fazer. 
As três respostas vencedoras foram escolhidas por Wollheim, autor da história, e Lowndes, editor da revista, e publicadas na edição de dezembro de 1941. Cada uma delas propunha uma solução diferente: o primeiro lugar sugeria que Janice matou apenas metade do mundo, o velho mundo. 0 segundo lugar falou que Janice matou toda a humanidade. Já o terceiro, assim como o primeiro, propôs que Janice matou metade da população, mas dessa vez a do novo mundo. Enquanto o segundo e terceiro lugares escreveram justificando suas escolhas, $o$ primeiro lugar colocou-se no papel de escritor e enviou o que seria a continuação da história.

Essa inversão de papéis mostra como leitores, autores e editores estavam próximos e como um fã poderia facilmente passar de leitor a autor - como é o caso dos próprios Futurians. Mas mais do que isso, mostra como era importante a participação dos leitores na construção da ficção científica. Quando os Futurians começaram a editar suas revistas, contaram com esse apoio. Na primeira edição como editor da Cosmic Stories, de março de 1941, o Futurian Donald Wollheim escreveu:

No matter how much theory or how much past experience one may think he has, it will never suffice to keep any magazine on the plane of quality and quantity its readers demand. That can only be done when readers do their part; when they write in their candid opinions of stories, articles, departments, art work and the editors, their suggestions as to what they would do if they were editor, their ideas (WOLLHEIM, 1941, p. 125).

Wollheim percebe e demonstra a importância que os leitores tem na ficção científica. A troca entre leitores, autores e editores moldou o gênero e ajudou a construir a ficção científica, com os Futurians fazendo parte e colaborando nesse movimento. Ao mesmo tempo em que a ficção científica era construída em seus temas e estruturas narrativas, ela também era pensada em seu formato. Em seus primeiros números, a Amazing Stories sobretudo republicou romances, serializados ao longo de vários números. Ao se firmar como pulp, escritores passaram a mandar seus textos também para a revista de Gernsback. Ainda que a Amazing e outras revistas que vieram na sequência continuassem publicando romances serializados, os formatos menores (contos, noveletas e novelas ${ }^{2}$ ) passarem a ser preferidos.

Na edição de janeiro de 1941 da pulp Super Science Stories, o Futurian Frederik Pohl, editor da revista desde seu lançamento, no ano anterior, anunciou uma mudança na revista:

Many of our readers have written in to this magazine and to its companion, Astonishing Stories, suggesting that a fault of modern science fiction lies in the fact that its authors are being driven to write progressively shorter and shorter stories. The responsibility for this trend can be traced to the plea of the readers for a wider variety of stories. The old styles of writing are changing; new concepts are coming to the fore. The incredible leaps that science itself has made open brand-new fields to explore (...).

A novel - to reduce the question to an equation - represents a very large investment of time and energy to its author. A bad novel - i.e., one which is rejected - represents a huge loss. Very naturally, an author will spend more time and trouble in putting the final, polishing touches on a novel

${ }^{2}$ A diferença, aqui, é em relação ao número de palavras: um conto tem até 7.500 palavras; noveleta de 7.500 a 17.500 ; novela, entre 17.500 e 40.000 palavras; e romance a partir de 40.000 palavras. 
than on a short story.

You readers have indicated that there is a place for a magazine specializing in well-written, well-developed, long stories. Accordingly, this is the last issue of Super Science Stories.

Its place will be taken in the future by Super Science Novels Magazine. It will present, each issue, either a - literally, not figuratively - book-lenghtnovel, plus one shorter novel, or three shorter novels. It will also include one or two short stories (POHL, 1941, p. 127 grifos no original).

As questões levantadas por Pohl mostram as vantagens de cada tipo de texto. Ainda que nos romances a trama possa ser melhor desenvolvida, a rapidez em escrever e publicar um conto é interessante quando um autor pretende explorar várias ideias diferentes. 0 pagamento também era um fator importante para esses escritores, pois as pulps costumavam pagar por palavra - quanto maior um texto, maior o cheque, mas também mais demorado e arriscado. Pohl decidiu testar esse novo formato para uma das revistas que editava: foram três edições em que ela passou a se chamar Super Science Novels e publicou romances, novelas e noveletas. Ele teve um desentendimento com o dono da editora e ficou afastado por alguns meses, e o novo editor voltou ao antigo formato e nome.

Os contos, de fato, dominavam as revistas pulp. Os autores de ficção científica da década de 1940 escreviam suas histórias sob medida para as revistas e algumas delas foram escritas para preencher "buracos" nas edições, o que permitiu que contos curtíssimos, de apenas poucas páginas fossem publicados. É o caso, por exemplo, do conto "Space Episode", da Futurian Leslie Perri, publicado na Future Combined with Science Fiction de dezembro de 1941, editada por Lowndes. Nele os astrounautas, Lida, Erik e Michael estão em um foguete que foi atingido por um cometa e alguém precisa desobstruí-lo, caso contrário os três morrerão. A pessoa que fizer isso, no entanto, sabe que morrerá, pois não conseguirá voltar para o interior do foguete. Erik e Michael ficam paralisados e é Lida quem assume a situação e se sacrifica.

"Space Episode" trouxe uma mulher astronauta para a ficção científica, algo muito raro à época e ainda fez dela a heroína da história, sacrificando-se para que os dois colegas pudessem voltar com segurança à Terra. 0 conto de Perri é hoje visto como uma peça importante na história da ficção científica feita por mulheres, já que o período é geralmente lembrado apenas pela participação masculina.

O comentário do crítico de ficção científica Paul A. Carter sobre o voo de Valentina Tereshkova mostra essa dimensão:

In 1963 the USSR's Valentina Tereshkova orbited the earth; from the standpoint of women's liberation in the West the shame of the USA's program was not so much that a woman pilot did not duplicate the feat as that our society did not feel that such a step was even imaginable. This specific kind of social history was something the magazine science fiction of the 1930s and 1940s rarely, if ever, foresaw (CARTER, 1977, p. 51).

De fato, a ficção científica dos anos 30 e 40 dificilmente retratava mulheres em situações de liderança - o conto de Perri é uma exceção. Não à toa, foi publicado em uma revista editada por um Futurian, que defendeu a história, atacada pela parcela masculina de leitores e muito elogiada pelas leitoras: 
More of you liked than disliked it. It seems as if a lot of masculine egos were wounded by Perri tale - but ardent feminists plugged it roundly. It served to show, if nothing else, that more than just a few girls read Future and they can be stirred into writing letters to the editor at times (LOWNDES, 1942a, p. 102).

O progressismo dos Futurians e sua preocupação com as questões sociais, permitiu que novas questões estivessem presentes nas páginas das revistas pulps. Em um longo comentário na edição de dezembro de 1942 da pulp Future Fantasy and Science Fiction, da qual também era editor, Lowndes escreve a respeito das preocupações que tinha sobre que tipo de história deveria publicar. Lowndes discute que por muitos anos os leitores de ficção científica foram considerados mais inteligentes do que os das outras revistas, mas que as histórias são acessíveis a todos, tenham um conhecimento mais avançado em ciências ou não. Esse comentário é muito interessante, pois tira a aura de excepcionalidade dos leitores de ficção científica e os coloca ao lado dos leitores de outras pulps. Essa visão é contrária, como aponta Lowndes, à noção que a ficção científica vinha construindo de si mesma até então, e que continuava sendo desenvolvida na revista "rival", a Astounding Science Fiction. Nela, os leitores de ficção científica eram considerados como uma "elite" (LUCKURST, 2005). Lowndes encerra o texto da seguinte maneira:

The ideal science-fiction story today is that tale so written as to afford entertainment to the passive reader, but to contain solid meat to within the framework of the story, and the ideas upon which the story is based, from which the active reader - he who not only reads words, but strives to ascertain what they mean, to discover what the writer of the words is trying to put over - can derive food for speculation. It is by no means easy to write this type of story, for which reason you'll find more of the strictly passive entertainment-type of fiction on the science fiction Rialto.

We'd like to hear comments from readers, agreeing or disagreeing upon the above opinions (LOWNDES, 1942b, p. 75).

Como editor, Lowndes tinha uma preocupação em atingir uma audiência maior do que apenas os fãs de ficção científica, mas ao mesmo tempo levar textos de qualidade, com várias camadas possíveis de interpretação. Como também era escritor, ele aponta que escrever esse tipo de texto não era fácil. Por fim, convida os leitores a enviarem suas opiniões sobre o assunto, demonstrando mais uma vez a importância dos leitores na construção da ficção científica.

\section{Considerações finais}

O cenário favorável ao mercado editorial das pulps de ficção científica, descrito acima, vai ser drasticamente alterado com a entrada dos Estados Unidos na Segunda Guerra Mundial. Em pouco tempo, o país impôs um racionamento de guerra de vários itens necessários para as revistas (como papel, tinta e tipos móveis de metal). Além disso, ficava cada vez mais difícil conseguir material para publicar, já que vários autores foram chamados para servir nas forças armadas. 0 número de revistas caiu drasticamente: "Most of the magazines survived 1942 and a few survived 1943, but few made it right through the war. By 1945 there were only 7 magazines compared with the peak of 22 in 
1941" (ASHLEY, 2000, p. 164). Das revistas editadas pelos Futurians, nenhuma sobreviveu à guerra.

0 grupo The Futurian Society of New York também teve fim em 1945 devido a desentendimentos internos. Inicialmente fãs, vários Futurians passaram a trabalhar profissionalmente com a ficção científica; segundo Damon Knight, o grupo deu origem a dez escritores, dois agentes literários, quatro antólogos e cinco editores - alguns com sobreposição de papéis (KNIGHT, 2013). 0 crítico Brian Attebery destaca a importância dos Futurians para a ficção científica:

One group, the Futurians, included many of the most important writers in the next generation: Frederik Pohl, Damon Knight, Judith Merril, Cyril Kornbluth, Isaac Asimov and James Blish. Three of those, Blish, Knight and Merril, also became important critics, pointing out logical flaws in sf stories and praising those writers who embodied scientific ideas in compelling narratives. Their efforts, and the willingness of fans to explore new fictional directions, helped transform the genre into something more sophisticated than its pulp beginnings (ATTEBERY, 2003, p. 38).

Segundo Attebery, os Futurians ajudaram a transformar o gênero. Testaram novas ideias, formas de escrita e tinham liberdade para produzir uma ficção científica diferente daquela que vinha sendo feita até então. Este artigo procurou mostrar algumas dessas contribuições dos Futurians à ficção científica, mas não pretende com isso de forma alguma esgotar o assunto, muito pelo contrário: este texto procurou servir como uma porta de entrada para os Futurians e sua imensa potência criativa.

\section{Referências}

ASHLEY, Mike. The Time Machines: The Story of the Science-Fiction Pulp Magazines from the Beginning to 1950. Liverpool: Liverpool University Press, 2000.

ASIMOV, Isaac. In Memory Yet Green: The Autobiography of Isaac Asimov, 1920-1954. New York: Doubeday, 1979 (e-book).

ATTEBERY, Brian. The magazine era: 1926-1960. In: JAMES, Edward; MENDLESOHN, Farah (org.). The Cambridge companion to science fiction. Cambridge: Cambridge University Press, 2003.

CARTER, Paul A. The creation of tomorrow: fifty years of magazine science fiction. New York: Columbia University Press, 1977.

CASHBAUGH, Sean. A Paradoxical, Discrepant, and Mutant Marxism: Imagining a Radical Science Fiction in the America Popular Front. Journal for the Study of Radicalism. East Lansing, v. 10, n.1, p. 63-106, 2016.

DAVIS, Blair. In: James Cameron's Story of Science Fiction. New York: AMC, 2018. Programa de TV.

KLIMARIS, J.S. Station X. Future Combined with Science Fiction, New York, v.2, n.2, p. 67, dez. 1941.

KNIGHT, Damon. The Futurians. Gollancz, 2013 (ebook).

LOWNDES, Robert. The Martians are Coming. Cosmic Stories. New York, v.1, n.1, p. 36-45, mar. 1941. 
Station X. Future Combined with Science Fiction. New York, v. 2, n. 4, p. 102-112, abr. 1942a.

Station X. Future Fantasy and Science Fiction. New York: v.3, n.2, p.75, dez. 1942b.

LUCKHURST, Roger. Science Fiction. Malden: Polity Press 2005.

MORLEY, Wilfred Owen [Robert Lowndes]. Science Fiction Classics. Future Fiction. New York: v.1, n.6, p.95, ago. 1941a.

Station X. Future Combined with Science Fiction Stories. New York, v.2, n.2, p. 68, dez. 1941b.

POHL, Frederik. An Important Announcement. Super Science Stories. New York: v.2, n.2, p. 127, jan. 1941.

The way the future was: a memoir. New York: Ballantine Books, 1978.

RICH, Mark. C.M. Kornbluth: The Life and Works of a Science Fiction Visionary. Jefferson: McFarland \& Company, 2009.

VINT, Sherryl. Science Fiction: A Guide for the Perplexed. New York: Bloomsbury, 2014.

WOLLHEIM, Donald. The Cosmoscope. Cosmic Stories. New York, v.1, n.1, p. 125, mar. 1941.

Artigo enviado em: 10/05/2021. Aprovado em: 05/07/2021. 